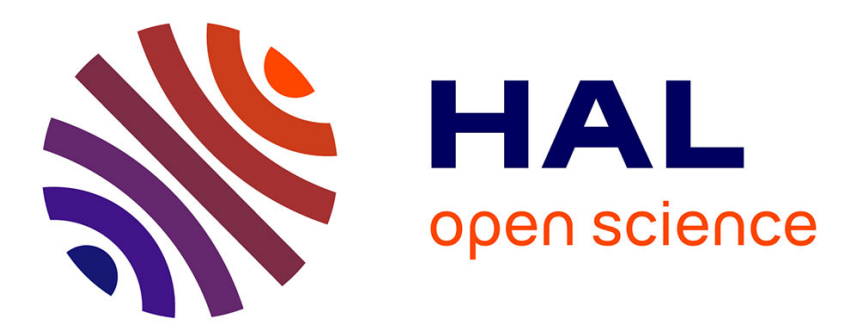

\title{
Efficient worst-case analysis of electronic networks in intervals of frequency
}

Moises Ferber de Vieira Lessa, Anton Korniienko, Johan Lofberg, Florent Morel, Gérard Scorletti, Christian Vollaire

\section{- To cite this version:}

Moises Ferber de Vieira Lessa, Anton Korniienko, Johan Lofberg, Florent Morel, Gérard Scorletti, et al.. Efficient worst-case analysis of electronic networks in intervals of frequency. International Journal of Numerical Modelling: Electronic Networks, Devices and Fields, 2017, pp.e2249. 10.1002/jnm.2249 . hal-01559420

\author{
HAL Id: hal-01559420 \\ https://hal.science/hal-01559420
}

Submitted on 18 Oct 2017

HAL is a multi-disciplinary open access archive for the deposit and dissemination of scientific research documents, whether they are published or not. The documents may come from teaching and research institutions in France or abroad, or from public or private research centers.
L'archive ouverte pluridisciplinaire HAL, est destinée au dépôt et à la diffusion de documents scientifiques de niveau recherche, publiés ou non, émanant des établissements d'enseignement et de recherche français ou étrangers, des laboratoires publics ou privés. 


\title{
Efficient Worst-case Analysis of Electronic Networks in Intervals of Frequency - copy
}

\author{
M. Ferber*, A. Korniienko, J. Löfberg, F. Morel, G. Scorletti, C. Vollaire \\ Federal University of Santa Catarina, Rua Dr. João Colin 2700, Sala E109a, 89.218-035 Joinville-SC, Brazil
}

\begin{abstract}
SUMMARY
This paper presents a new method to compute upper and lower bounds of any voltage or current of an arbitrary linear electric circuit model with uncertain parameters. The bounds are in the frequency domain and when compared to a previously proposed method, this novel approach provides a higher level of guarantee. The reason is that the bounds are not only computed for a set of fixed frequencies, but computed to a set of intervals of frequencies. The details of the proposed approach, especially the equivalent uncertain element models, are given. Additionally, tests are carried out on problems with low and high number of uncertain parameters. Contrary to the classical method of Monte Carlo, the results are not based on a random choice of parameters and do not depend on the number of iterations. It is shown on an example that the classical method of Monte Carlo needs a high number of iterations in order to reach results in agreement with the proposed method. Then, it leads to higher computation times of several orders of magnitude.
\end{abstract}

KEY WORDS: Worst-case Analysis, Robustness Analysis, Electric Circuits, Convex Optimization, Uncertainty

\section{INTRODUCTION}

Efficient engineering design relies heavily on prediction by accurate models of real systems, subsystems and components. Today, there are several software packages available to model and simulate engineering problems with a high degree of accuracy. Some examples of these software packages are Simulink [1], Modelica [2] and ANSYS [3]. Usually, these software can deal with very complex multiphysics problems that engineers face today, which contains several input and output variables, several physical parameters and may be considered in the time- or frequency-domain.

In reality, the system to be modelled has often some kind of uncertainty which cannot be neglected if one desires to build a reliable model [4]. For example, in electrical engineering, phenomena such as production dispersion, ageing, unknown operational temperature and humidity, and external interference may significantly influence the behavior of electronic circuits. Therefore, a model must take all these uncertainty into account in order to be considered accurate and reliable. The question now is: how to guarantee a given level of performance in spite of uncertainties?

*Correspondence to: Federal University of Santa Catarina, Rua Dr. João Colin 2700, Sala E109a, 89.218-035 JoinvilleSC, Brazil 
There has been a lot of effort from the scientific community to tackle the problem of uncertainty analysis in electrical engineering, as it can be verified by the growing number of publications in this field [5-20].

The first widely used method for uncertainty quantification is the Monte Carlo method [5] and it was first used by researchers at Los Alamos Laboratory, during the development of the atomic bomb. This method consists of carrying out several deterministic simulations of a problem, varying the uncertain parameter values on each simulation. In this manner, one attempts to cover all possible scenarios in which the system may operate. This method can therefore be applied to almost any problem: linear and non-linear, in the time- and frequency-domain, and with few or many uncertain parameters. Because of its generality, the Monte Carlo method is usually available in typical engineering simulation software packages as a tool for uncertainty analysis.

In [6], the method of Monte Carlo was utilised for the uncertainty analysis of noise-parameters of transistors and in [7] the Monte Carlo method was used for uncertainty analysis of a commercial microwave noise temperature measurement.

There is, however, an important disadvantage of the Monte Carlo method: it may require an unpractical high number of simulations, $10^{4}$ or more [8], in order to cover well the uncertainty space and consequently obtain an accurate result. In applications where one simulation takes minutes, hours or even days to complete, this method would require years or decades to complete a computation.

In this context, several research papers presenting alternative methods to the Monte Carlo method have been published. In [9], the author considered only the uncertainty analysis problem of approximate linear models, where the output is related to the uncertain parameters only by a multiplier or a gain. In this case, it is straightforward to obtain results of average and standard deviation, since there are analytical expressions available. Therefore, the interest of this method is very limited.

In $[10,11]$, the Unscented Transform (UT) was applied to compute the average and the standard deviation of an output, given an electromagnetic problem with uncertain parameters. The UT is an excellent alternative method to the Monte Carlo one, given that the goal of the designer is to determine statistical moments of an output and that the problem does not have more than a few uncertain parameters. Indeed, the UT suffers from the curse of dimensionality, since its computational complexity is exponential with the number of uncertain parameters. Additionally, when one applies the Unscented Transform to a problem, one does not obtain information on the bounds of the output variable, but only about the average and its vicinity.

In [12], an alternative collocation method called Stroud was proposed to reduce the computational complexity of the UT. Even though the proposed method has a linear or quadratic computational complexity with the number of uncertain parameters, its accuracy is limited and no information of bounds can be assessed. In [13], another collocation method is proposed and applied to an electrical engineering problem, but no solution to the curse of dimensionality is presented.

In [14], the author proposes a method based on interval arithmetics to deal with the uncertainty. This is an interesting approach, again when the problem has only a few uncertain parameters. This method tends to produce conservative results and it cannot be generalized to large and relevant problems. Another two methods based on interval arithmetics are presented in $[15,16]$, but again the computational complexity subject is left aside. 
In [17], an approximate solution to the curse of dimensionality is proposed. This solution is based on the fact that most often only a subset of the uncertain parameters may actually influence the output variable. Thus, a pre-processing is carried out on the physical model to screen unnecessary input variables from the original model.

Some other papers report using a combination of genetic algorithms and affine arithmetics [18] to find bounds of an output variable of interest. This approximate approach is based on a stochastic method which has no guarantee of convergence in the general case and no study has shown that it is more efficient than pure Monte Carlo. In [19], an interesting and new approach based on wavelets is proposed, but again the lack of formality does not guarantee reliable bounds to the problem addressed in this paper.

Finally, in [20], a completely different approach to the uncertainty analysis problem has been proposed to the electrical engineering community. This proposed approach is well-suited to uncertain linear circuit models in the frequency-domain and it focuses on the worst-case tolerance analysis. It is based on convex optimization, and not on stochastic optimization, thus it has some very interesting advantages over the Monte Carlo method. First, the convergence of the computation of the upper and lower bounds on the frequency response of electronic networks is guaranteed to be achieved. Second, in order to obtain comparable results, the Monte Carlo method needs so many iterations that it needs a computation time longer by several orders of magnitude than the time needed to achieve the convergence of the proposed method. Finally, it is a formal method with a strong foundation theory developed by the Robust Control community.

In this paper, we propose an alternative modeling technique, in the context of [20], in order to compute upper and lower bounds of the frequency response of uncertain electronic networks. Here, we use intervals of frequency instead of fixed values of frequency. This characteristic can speed up even more the uncertainty analysis when compared to the Monte Carlo method or to the method proposed in [20]. Moreover, the proposed approach avoids missing low-damped resonances, by choosing appropriate ranges of frequency. The theoretical background discussion can be found in [21].

The remainder of this paper is organized as follows: in section 2 the problem statement is presented and in section 3 the modelling methodology to incorporate the frequency as an uncertain parameter is given. Then, in section 4, two application cases are depicted while in section 5 numerical results are presented and confronted with the Monte Carlo method. Finally, in section 6, a conclusion is given discussing the main advantages and disadvantages of the proposed method. The paper ends with the acknowledgements to the funding agencies supporting this work.

\section{PROBLEM STATEMENT}

Consider an arbitrary electric circuit, composed of linear components such as resistors, inductors and capacitors. Each value of these components is a parameter of the electric circuit model and it is denoted by $p_{k}$. The parameter values may be subject to uncertainty and thus they are only known to belong to an interval $p_{k} \in\left[p_{k}^{\min }, p_{k}^{\max }\right]$. The input and output of the circuit may be any voltage or current associated with the components and they are denoted $w_{p}$ and $z_{p}$, respectively. The transfer 
function between $w_{p}$ and $z_{p}$ is $T_{w_{p} \rightarrow z_{p}}(s)$ which is defined as:

$$
T_{w_{p} \rightarrow z_{p}}(s)=\frac{\sum_{j=0}^{m} b_{j}(p) s^{j}}{\sum_{i=0}^{n} a_{i}(p) s^{i}},
$$

where $p=\left(p_{1}, \cdots, p_{N}\right)$ the vector of the $N$ parameters of the linear electrical circuit, $a_{i}$ and $b_{j}$ are the transfer function coefficients, in general rational functions of the parameters regrouped in $p$.

Two problems are addressed by this paper and they consist of determining upper and lower bounds in the frequency domain of $\left|T_{w_{p} \rightarrow z_{p}}(j \omega)\right|$. The mathematical formulation of these problems are given in the two subsections below.

\subsection{Worst-case upper bound problem}

Given $T_{w_{p} \rightarrow z_{p}}(s), p_{k}^{\min }$ and $p_{k}^{\max }$, frequency intervals $\left[\omega_{l}^{\min }, \omega_{l}^{\max }\right]$, compute the smallest $\eta_{l}$ such that for any $p_{k} \in\left[p_{k}^{\min }, p_{k}^{\max }\right]$ and any $\bar{\omega} \in\left[\omega_{l}^{\min }, \omega_{l}^{\max }\right]$, for all $k$ and $l$

$$
\left|T_{w_{p} \rightarrow z_{p}}(j \bar{\omega})\right| \leq \eta_{l}
$$

\subsection{Worst-case lower bound problem}

Given $T_{w_{p} \rightarrow z_{p}}(s), p_{k}^{\min }$ and $p_{k}^{\max }$, frequency intervals $\left[\omega_{l}^{\min }, \omega_{l}^{\max }\right]$, compute the largest $\beta_{l}$ such that for any $p_{k} \in\left[p_{k}^{\text {min }}, p_{k}^{\text {max }}\right]$ and any $\bar{\omega} \in\left[\omega_{l}^{\text {min }}, \omega_{l}^{\max }\right]$, for all $k$ and $l$

$$
\beta_{l} \leq\left|T_{w_{p} \rightarrow z_{p}}(j \bar{\omega})\right|
$$

\section{METHODOLOGY}

\subsection{Theoretical background}

The methodology proposed here to solve the problems stated in section 2 is an extended version of the method proposed in our recent work [20]. As discussed before, the method from [20] allows one to compute the bounds of the frequency response similar to (2) and (3) but only for a given frequency $\omega$. It is then possible to consider a frequency grid and solve the problem for each frequency. However, this method will not ensure the bounds in-between the chosen frequency points and, additionally, an appropriate choice of the grid could be a tricky problem, especially for complex circuits. For this reason, in this paper, the bounds are computed not for a given frequency $\omega$ but for a given range of frequencies $\left[\omega_{l}^{\min }, \omega_{l}^{\max }\right]$. Hence, considering a grid on values of $\omega_{l}^{\min }$ and $\omega_{l}^{\max }$ such that the frequency ranges overlap each other will ensure an appropriate bounds computation. From now only one frequency range is thus under consideration, i.e. $\left[\omega_{l}^{\min }, \omega_{l}^{\max }\right]$ and $l$ is fixed. The key idea is to represent the frequency as an uncertain parameter, just as it is done with uncertain physical parameters. In this manner, the frequency lies within the range $\left[\omega_{l}^{\min }, \omega_{l}^{\max }\right]$. 
According to [20], the uncertain parameters can be normalized and the transfer function $T_{w_{p} \rightarrow z_{p}}(s)$ becomes:

$$
T_{w_{p} \rightarrow z_{p}}(s)=\frac{\sum_{j=0}^{m} \widehat{b}_{j}(\delta) s^{j}}{\sum_{i=0}^{n} \widehat{a}_{i}(\delta) s^{i}}
$$

where $\widehat{a}_{i}(\delta)$ and $\widehat{b}_{j}(\delta)$ are the transformed coefficients, rationally dependent functions of normalized parameter vector $\delta=\left[\delta_{1}, \cdots, \delta_{N}\right]$ with $\left|\delta_{k}\right| \leq 1$, for all $k$. Basically, the uncertain parameter vector $p$ is replaced by a normalized one $\delta$.

Let us now introduce the following change of variables

$$
\bar{\omega}=\omega\left(1+\delta_{\omega} \omega_{g}\right)
$$

with $\omega=\left(\omega_{l}^{\max }+\omega_{l}^{\min }\right) / 2$ and $\omega_{g}=\frac{\omega_{l}^{\max }-\omega_{l}^{\min }}{\omega_{l}^{\max }+\omega_{l}^{\min }}$. Please note that, since $\bar{\omega} \in\left[\omega_{l}^{\min }, \omega_{l}^{\max }\right], \delta_{\omega}$ is normalized uncertain frequency parameter: $\delta_{\omega} \in[-1,+1]$. Then, similar to [20], a Linear Fractional Transformation (LFT, see [22]) representation of the system, can be associated to the electrical circuit. It will be denoted as $\Delta \star M$ where $\Delta$ is uncertain part of the circuit and $M$ is its nominal part. The uncertain frequency response of the circuit, $T_{w_{p} \rightarrow z_{p}}(j \bar{\omega})$, for all $\bar{\omega} \in\left[\omega_{l}^{\text {min }}, \omega_{l}^{\text {max }}\right]$ can thus be evaluated in frequency domain by:

$$
\left[\begin{array}{c}
z(j \omega) \\
z_{p}(j \omega)
\end{array}\right]=M(j \omega)\left[\begin{array}{c}
w(j \omega) \\
w_{p}(j \omega)
\end{array}\right] \text { and } w(j \omega)=\Delta z(j \omega)
$$

where $\Delta$ is defined by

$$
\Delta=\left[\begin{array}{c|ccc}
\delta_{\omega} I_{n_{\omega}} & 0 & 0 & 0 \\
\hline 0 & \delta_{1} I_{n_{1}} & 0 & 0 \\
0 & 0 & \ddots & 0 \\
0 & 0 & 0 & \delta_{N} I_{n_{N}}
\end{array}\right]
$$

with $\left|\delta_{\omega}\right| \leq 1,\left|\delta_{k}\right| \leq 1$ for all $k, n_{\omega}$ the order of the system (number of dynamical components) and $n_{k}$ defining the number of repetitions of uncertain parameter $\delta_{k}$ in the uncertain circuit model.

Thanks to a rather straightforward application of the result [20,21], the solution of the worst-case upper bound problem is found by solving the following optimization problem:

$$
\begin{aligned}
& \min _{\eta^{2}, \Phi} \eta^{2} \\
& \text { subject to } \\
& {\left[\begin{array}{c}
M \\
I
\end{array}\right]^{*}\left[\begin{array}{cccc}
\Phi_{11} & 0 & \Phi_{12} & 0 \\
0 & I_{n_{z}} & 0 & 0 \\
\Phi_{21} & 0 & \Phi_{22} & 0 \\
0 & 0 & 0 & -\eta^{2} I_{n_{w}}
\end{array}\right]\left[\begin{array}{c}
M \\
I
\end{array}\right] \leq-\epsilon I}
\end{aligned}
$$


with $\epsilon>0$, and $\Phi=\left[\begin{array}{c||c}\Phi_{11} & \Phi_{12} \\ \hline \hline \Phi_{21} & \Phi_{22}\end{array}\right] \in \underline{\Phi}$ where the parametrization set $\underline{\Phi}$ is defined, accordingly to uncertainty structure (6), as:

$$
\Phi=\left\{\Phi \mid \Phi=\left[\begin{array}{c|ccc||c|ccc}
D_{\omega} & 0 & \cdots & 0 & G_{\omega} & 0 & \cdots & 0 \\
\hline 0 & D_{1} & 0 & \cdots & 0 & G_{1} & \cdots & 0 \\
\vdots & & \ddots & & & & \ddots & \\
0 & \ldots & 0 & D_{N} & 0 & \ldots & 0 & G_{N} \\
\hline \hline-G_{\omega} & 0 & \cdots & 0 & -D_{\omega} & 0 & \cdots & 0 \\
\hline 0 & -G_{1} & 0 & \cdots & 0 & -D_{1} & \cdots & 0 \\
\vdots & & \ddots & & & & \ddots & \\
0 & \ldots & 0 & -G_{N} & 0 & \cdots & 0 & -D_{N}
\end{array}\right]\right\}
$$

with $D_{\omega}, G_{\omega}$ and $D_{k}, G_{k}$ are complex, full, respectively $n_{\omega} \times n_{\omega}$ and $n_{k} \times n_{k}$ hermitian and skew hermitian frequency depending matrices i.e. $D_{\omega}^{*}=D_{\omega}, D_{k}^{*}=D_{k}$ and $G_{\omega}^{*}=-G_{\omega}, G_{k}^{*}=-G_{k}$. They represent the decision variables of the optimization problem (7), see [20,21] for more details or $\mu$-analysis interpretation of this result in [26,27]. The optimization problem (7) is a convex LMI optimization problem and thus it can be solved efficiently. Furthermore, since the worst-case lower bound problem (2) can be transformed to an equivalent worst-case upper bound problem, both problems considered in this paper can be efficiently solved by (7) provided an LFT representation of electrical circuit (5) and (6) is found. The next subsection provides a systematic LFT derivation procedure in the context of electrical circuits, allowing to take into account the frequency uncertainty.

\subsection{Systematic LFT Derivation with frequency uncertainty}

First, let us consider the relation between voltage and current of an uncertain inductor in the frequency domain, since the proposed method operates directly in the frequency domain. This relation is given in (9), considering an uncertain frequency.

$$
V_{L}^{\text {tot }}(j \omega)=j \underbrace{L_{0}\left(1+L_{g} \delta_{L}\right)}_{\text {Uncertain value }} \underbrace{\left(1+\omega_{g} \delta_{\omega}\right) \omega}_{\text {Uncertain frequency }} I_{L}(j \omega)
$$

Then, the expression of an equivalent inductor, containing both parametric and frequency uncertainty, is depicted in (10).

$$
L=L_{0}\left(1+\delta_{L} L_{g}\right)\left(1+\delta_{\omega} \omega_{g}\right)
$$

After some analytic manipulations, it is possible to expand the initial expression into three terms in the time domain. Each term corresponds to an element of the inductor's equivalent circuit, shown in Fig. 1. The derivation is given as follows. 


$$
\begin{gathered}
v_{L}^{t o t}=L_{0}\left(1+L_{g} \delta_{L}\right)\left(1+\omega_{g} \delta_{\omega}\right) \frac{d i_{L}}{d t} \\
v_{L}^{t o t}=L_{0}\left(1+L_{g} \delta_{L}+\omega_{g} \delta_{\omega}+L_{g} \delta_{L} \omega_{g} \delta_{\omega}\right) \frac{d i_{L}}{d t} \\
v_{L}^{t o t}=L_{0} \frac{d i_{L}}{d t}+L_{0} \frac{d i_{L}}{d t} L_{g} \delta_{L}+\left(L_{0} \frac{d i_{L}}{d t}+L_{0} \frac{d i_{L}}{d t} L_{g} \delta_{L}\right) \omega_{g} \delta_{\omega}
\end{gathered}
$$

Note that in contrast to the equivalent circuit proposed in [20], an additional term, related to the uncertain frequency, is added.

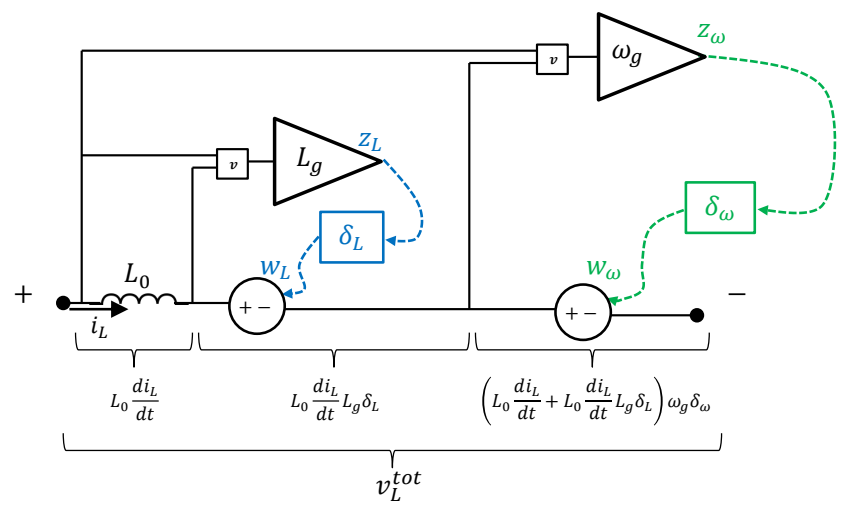

Figure 1. Uncertain Inductor equivalent schematic

A similar procedure can be used to derive the equivalent circuit of the capacitor with both parametric and frequency uncertainty, which leads to expressions (11) and (12) and to Fig. 2.

$$
\begin{gathered}
C=C_{0}\left(1+\delta_{C} C_{g}\right)\left(1+\delta_{\omega} \omega_{g}\right) \\
i_{C}^{t o t}=C_{0} \frac{d v_{C}}{d t}+C_{0} \frac{d v_{C}}{d t} C_{g} \delta_{C}+\left(C_{0} \frac{d v_{L}}{d t}+C_{0} \frac{d v_{C}}{d t} C_{g} \delta_{C}\right) \omega_{g} \delta_{\omega}
\end{gathered}
$$

The uncertain components have terminals for the electric connections as well as terminals for the uncertainty connections. The electric connections define the circuit topology whereas the uncertainty connections define the LFT representation of the system (5) and (6). Once the circuit is completely built, with all the electric and uncertainty connections defined, the LFT is obtained by extraction of a continuous-time linear state-space model around the defined operating point [25]. In this LFT model, each uncertain parameter (physical and frequency) are associated with an input and an output variable.

In Fig. 3, an example with a RLC circuit is given. Note that the input is a voltage source and the output is a voltage measurement. Additionally, there are the electric connections forming the circuit topology and the uncertain connections constituting the LFT representation with one variable per 


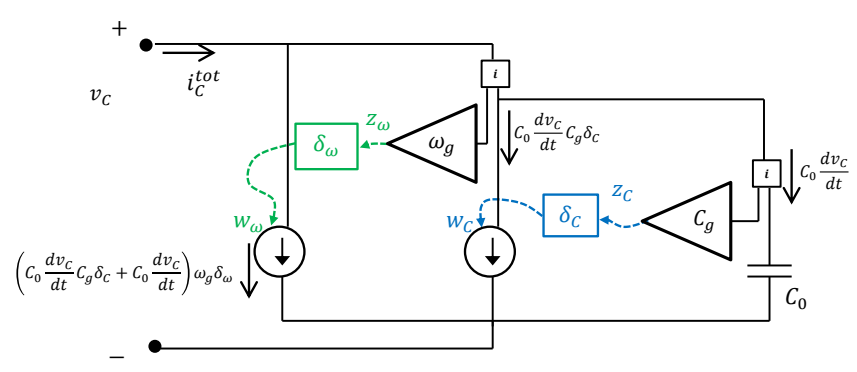

Figure 2. Uncertain Capacitor equivalent schematic

uncertain parameter. Finally, note that each dynamical component (the inductor and the capacitor, but not the resistor) gives rise to a frequency uncertainty variable.

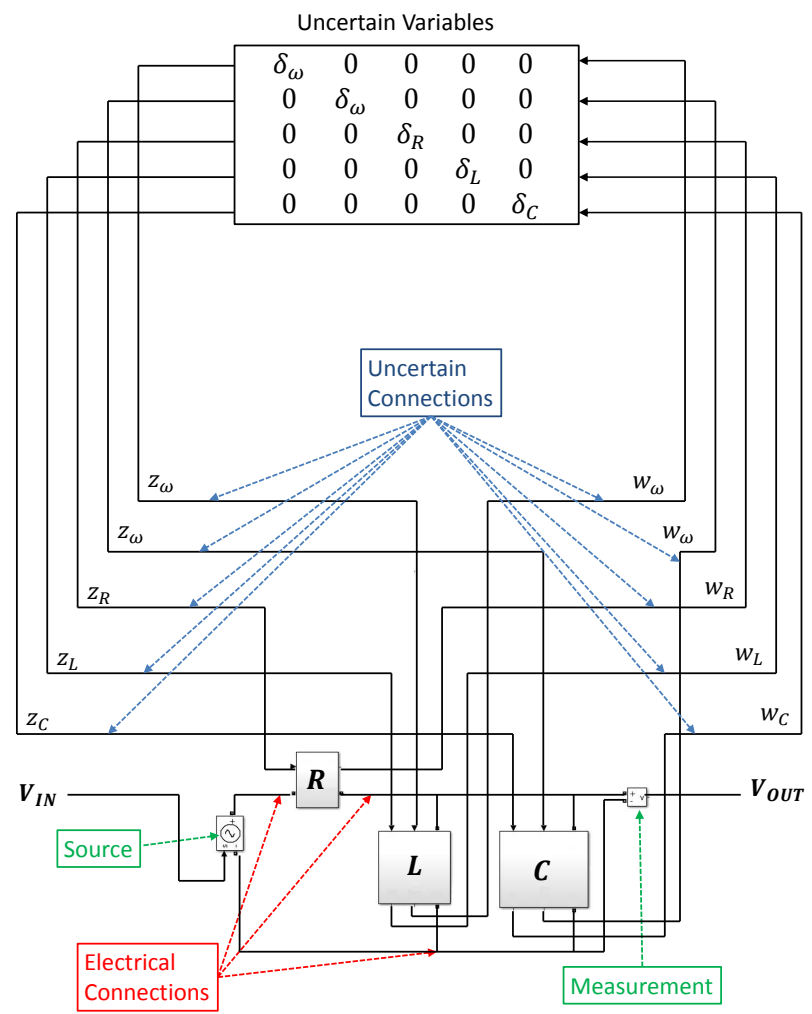

Figure 3. Systematic extraction of LFT representation

It is important to emphasize that the frequency uncertainty variable $\delta_{\omega}$ is the same variable for all uncertain dynamical components (see equations (10) and (11)). Thus, in Fig. 3, the same frequency variable is repeated twice. In general, when $N$ dynamical components are present in the model, the $\delta_{\omega}$ appears $N$ times.

The nominal part of the LFT representation, $M$, allows the prompt application of a convex optimization (7), as described in details in [20]. The proposed method computes bounds that are similar to a stairway. The width of the steps can be adjusted by the uncertainty frequency parameter. 
The wider the frequency uncertainty is, the faster is the total computational speed - for less runs of the optimization solver are necessary to cover the entire frequency range. The cost of computing the bounds faster is a more conservative estimate of the results.

As the frequency uncertainty tends to zero, the results of the proposed method tend to be the same as the results obtained in [20].

\section{APPLICATION}

In this work, we have used the optimization language YALMIP [23] and the solver MOSEk [24] as the main tools to solve the optimization problem (7) and thus problems (2) and (3).

The proposed methodology was applied to two cases: an RLC circuit and a model of a transmission line with four RLRC cells. The first application is quite simple and it serves as an illustration of the method. The second application is more complex and it shows how interesting is the proposed method when compared to the Monte Carlo method.

\subsection{Case 1}

The application case 1 is an RLC circuit illustrated in Fig. 4. The input is a voltage source and the output is a voltage measurement. The uncertain parameters are the resistance, inductance and capacitance of the electrical components and their values are given in Table I. The overall frequency range considered in this application case is from $10^{4}$ to $10^{6} \mathrm{~Hz}$, with 10 intervals logarithmically spaced.

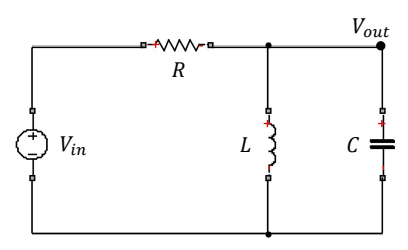

Figure 4. Application 1

Table I. Component Values of Application 1

\begin{tabular}{c|ccc} 
Parameter & Nominal & Minimum & Maximum \\
\hline $\mathrm{R}$ & $6000 \Omega$ & $3000 \Omega$ & $9000 \Omega$ \\
$\mathrm{L}$ & $1 \mathrm{mH}$ & $0.5 \mathrm{mH}$ & $1.5 \mathrm{mH}$ \\
$\mathrm{C}$ & $1 \mathrm{nF}$ & $0.5 \mathrm{nF}$ & $1.5 n \mathrm{~F}$
\end{tabular}

The uncertain equivalent circuit associated with the topology given in Fig. 4 is presented in Fig. 5. The nominal part of the LFT representation $M$ associated with this application case is a square matrix of dimension 6 . Two dimensions are related to the frequency uncertainty, three dimensions are related to the parametric uncertainty and one dimension is related to the transfer function from the input to the output voltage. 


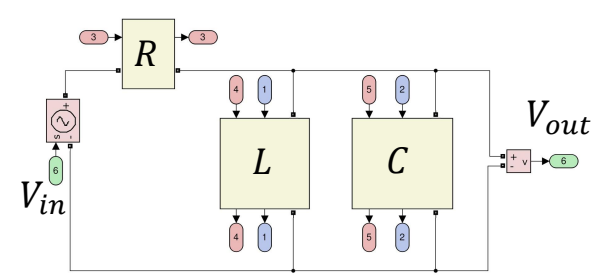

Figure 5. Application 1

\subsection{Case 2}

The application case 2 is a model of a transmission line containing four RLRC cells illustrated in Fig. 6. The input $\left(V_{i n}\right)$ is a voltage source and the output $\left(V_{\text {out }}\right)$ is a voltage measurement. The uncertain parameters are the resistances, inductances and capacitances of the electrical components and their values are given in Table II. The frequency range considered in this application case is from $10^{4}$ to $10^{8} \mathrm{~Hz}$, with 20 intervals logarithmically spaced.

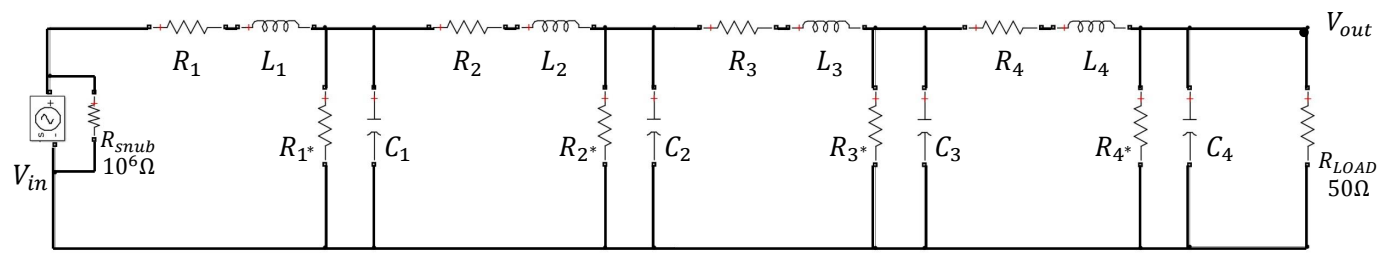

Figure 6. Application 2

Table II. Component Values of Application 2, where $k$ ranges from 1 to 4

\begin{tabular}{c|ccc} 
Component & Nominal & Minimum & Maximum \\
\hline$R_{k}$ & $0.1 \Omega$ & $0.05 \Omega$ & $0.15 \Omega$ \\
$L_{k}$ & $1 \mu H$ & $0.5 \mu H$ & $1.5 \mu H$ \\
$R_{k *}$ & $500 k \Omega$ & $250 k \Omega$ & $750 k \Omega$ \\
$C_{k}$ & $1 n F$ & $0.5 n F$ & $1.5 n F$
\end{tabular}

The uncertain equivalent circuit associated with the topology given in Fig. 6 is presented in Fig. 7. The nominal part of the LFT representation $M$ associated with this application case is a square matrix of dimension 25. Eight dimensions are related to the frequency uncertainty, sixteen dimensions are related to the parametric uncertainty and one dimension is related to the transfer function from the input to the output voltage.

\section{RESULTS}

\subsection{Case 1 - RLC Circuit}

The results of the application case 1 are shown in Fig. 8. 


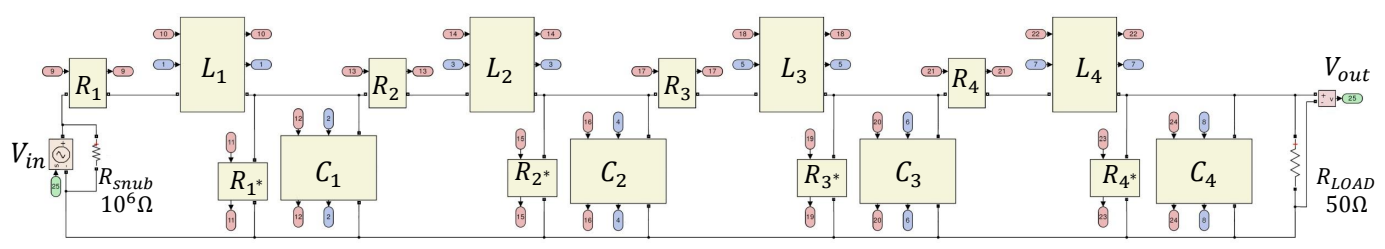

Figure 7. Application 2

Note that the results of some samples at the boundaries of the uncertainty parameter space are actually under and over the Monte Carlo lower bound and upper bound, respectively. This means that 1000 samples, in this experiment, were not sufficient to compute a reliable lower bound. Thus, Fig. 8 shows that it may be necessary many more random samples to achieve reliable results such as the proposed method. This fact motivates the relevance of the proposed approach.

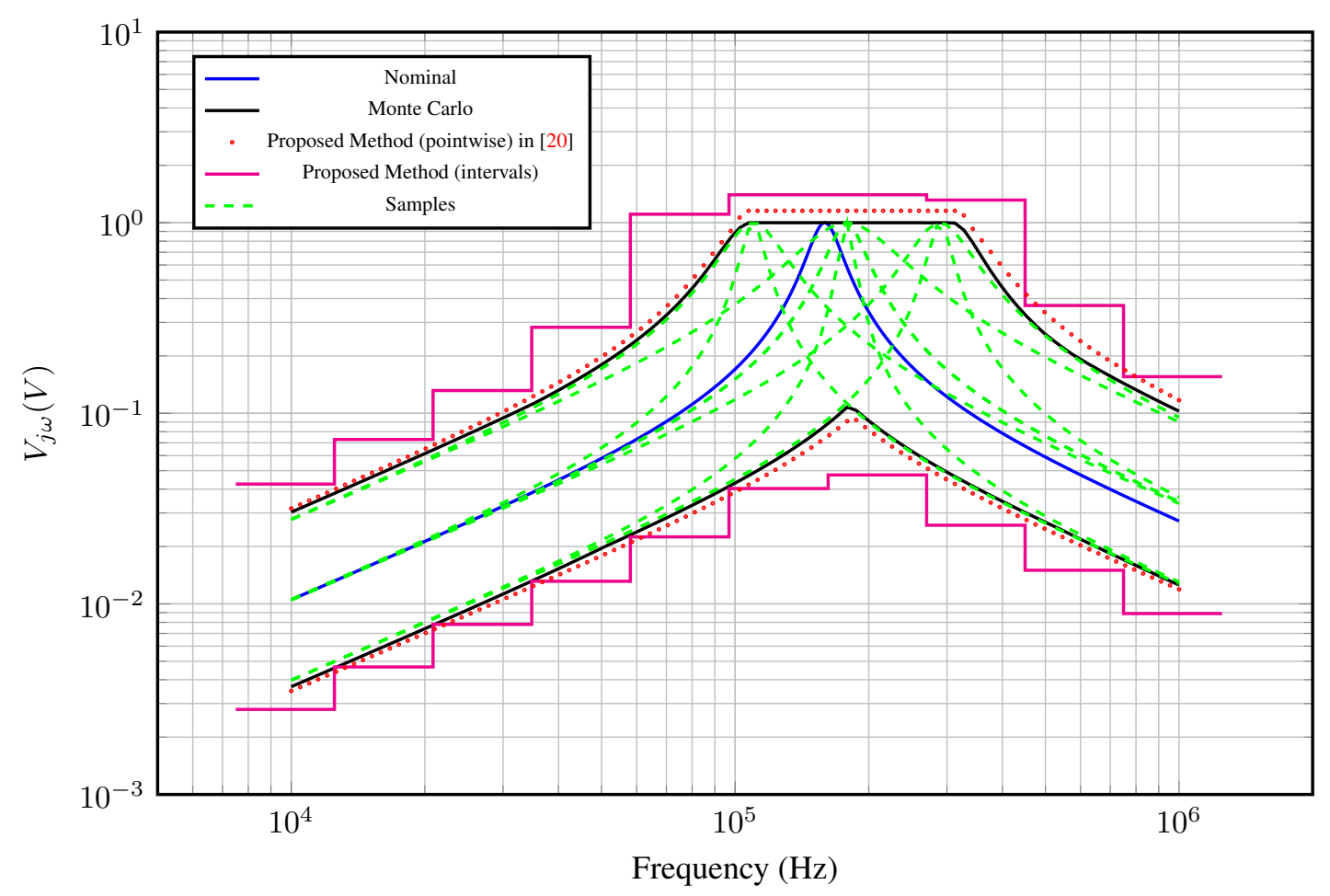

Figure 8. Results of the RLC circuit. In blue is the nominal response of the system, in black are the upper and lower bounds computed with the Monte Carlo method $\left(10^{3}\right.$ samples), in light green are some samples of the system response chosen at the boundaries of the uncertain parameter space, in red are the upper and lower bounds computed with the previously proposed method in [20] and in magenta are the upper and lower bounds computed with the proposed method in this paper.

The time required to carry out the Monte Carlo method, the proposed method in [20] and the proposed method here was approximately $3.84 \mathrm{~s}, 0.017 \mathrm{~s}$ and $0.025 \mathrm{~s}$ respectively. Both methods recently developed by the authors showed a speed increase of a factor over 100 . The proposed method here required a slightly longer computational time, which is expected. The proposed method here operated with a matrix of size 6 whereas the method proposed in [20] operated with a matrix of 
size 4. The increased size of the matrix allows for the consideration of uncertainty in the frequency domain, as explained previously. These extra dimensions allow a more guaranteed upper and lower bounds computation.

\subsection{Case 2 - Transmission Line model}

The results of the application case 2 are shown in Fig. 9.

Note that the results of some samples at the boundaries of the uncertainty parameter space are actually under the Monte Carlo lower bound. This means that 1000 samples, in this experiment, were not sufficient to compute a reliable lower bound. Thus, Fig. 9 shows that it may be necessary many more random samples to achieve reliable results such as the proposed method. Again, this fact motivates the relevance of the proposed approach.

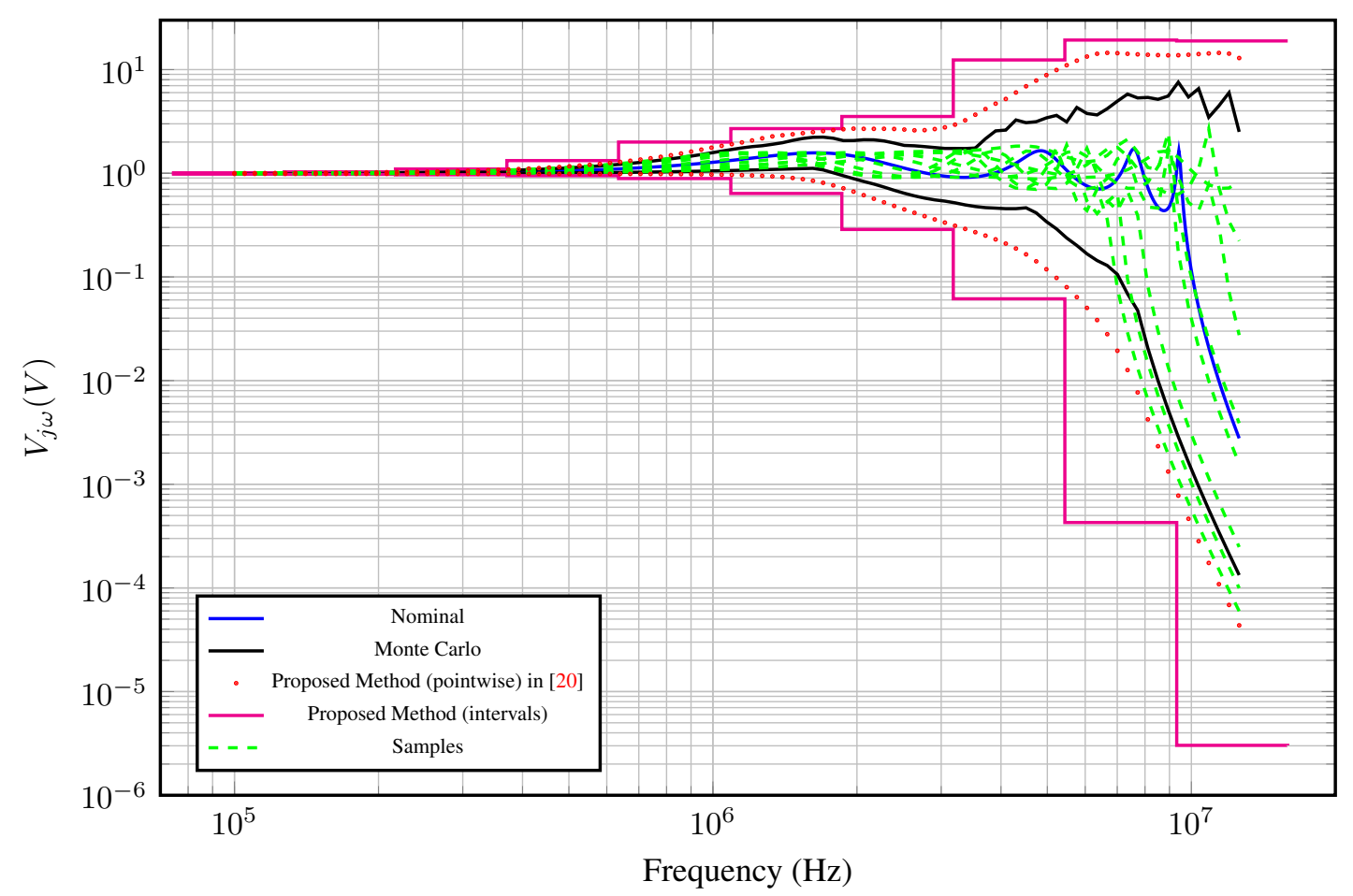

Figure 9. Results of the transmission line model, in blue is the nominal response of the system, in black are the upper and lower bounds computed with the Monte Carlo method ( $10^{3}$ samples), in light green are some samples of the system response chosen at the boundaries of the uncertain parameter space, in red is the upper and lower bounds computed with the previously proposed method [20] and in magenta is the upper and lower bounds computed with the method proposed in this paper.

The time required to carry out the Monte Carlo method, the proposed method in [20] and the proposed method here was approximately $1119.41 \mathrm{~s}$ (18.66min), $0.18 \mathrm{~s}$ and $1.45 \mathrm{~s}$ respectively. Both methods recently developed by the authors showed a speed increase of a factor over 1000. Even though the proposed method in [20] is approximately 10 times faster than the proposed method here, the later produces more guaranteed upper and lower bounds. 


\section{CONCLUSION}

This paper presented an actual and relevant problem in electrical engineering, which is the worstcase tolerance analysis of electronic networks. This problem is in the context of robustness analysis and it is an important area of research in computer-aided analysis and design.

A formal method, which is proven to be efficient, was adapted from the Robust Control theory and presented to solve the proposed problem. This method is based on convex optimization and its computational complexity is polynomial with the number of uncertain parameters. Therefore, its convergence is much faster than the Monte Carlo Method.

The proposed method was applied to two circuits. First, it was applied to a simple RLC circuit in order to better illustrate the application of the method. Then, it was applied to a model of a transmission line, containing four RLRC cells. The results obtained in both cases were in agreement to the expected results. Moreover, the proposed method completed its computation much earlier than the Monte Carlo method and additionally it produced more reliable results.

\section{ACKNOWLEDGEMENTS}

This work was partly supported by the LIA Maxwell French-Brazilian collaboration and partly by a grant from the Swedish-Brazilian Research and Inovation Center (CISB).

\section{REFERENCES}

1. Online: http://www.mathworks.com/products/simulink/.

2. Online: https://www.modelica.org/.

3. Online: http://www.ansys.com/.

4. D. H. Mac, S. Clenet, K. Beddek, L. Chevallier, J. Korecki, O. Moreau and P. Thomas, "Influence of uncertainties on the B(H) curves on the flux linkage of a turboalternator," in Int. J. Numer. Model. 2014; 27:385-399.

5. Nicholas Metropolis and S. Ulam, "The Monte Carlo Method," in Journal of the American Statistical Association, vol. 44, n. 247, pp. 335-341, 1949.

6. James Randa, "Numerical modeling and uncertainty analysis of transistor noise-parameter measurements," in Int. J. Numer. Model. 2015; 28:628-638.

7. Mark H. Weatherspoon and R. Joe Smith, "Uncertainty analysis of a commercial microwave noise temperature measurement system using Monte Carlo simulations," in Int. J. Numer. Model. 2011; 24:13-23

8. Ferber, M.; Vollaire, C.; Krahenbuhl, L.; Coulomb, J.-L.; Vasconcelos, J. A., ”Conducted EMI of DC-DC Converters With Parametric Uncertainties," in IEEE Transactions on Electromagnetic Compatibility (Print), vol.55, iss.4, p.699706, 2013.

9. E. Garcia, "Electromagnetic compatibility uncertainty, risk, and margin management," Electromagnetic Compatibility, IEEE Transactions on, vol. 52, no. 1, pp. 3-10, Feb 2010.

10. L. de Menezes, A. Ajayi, C. Christopoulos, P. Sewell, and G. Borges, "Efficient computation of stochastic electromagnetic problems using unscented transforms," Science, Measurement Technology, IET, vol. 2, no. 2, pp. 88-95, march 2008.

11. G. A. Borges, E. A. C. Junior and L. R. A. X. de Menezes, "Algorithms for uncertainty propagation in transmissionline matrix (TLM) method," in Int. J. Numer. Model. 2007; 21:43-60.

12. H. Bagci, A. Yucel, J. Hesthaven, and E. Michielssen, "A fast stroud-based collocation method for statistically characterizing emi/emc phenomena on complex platforms," Electromagnetic Compatibility, IEEE Transactions on, vol. 51, no. 2, pp. 301-311, May 2009. 
13. A. Yucel, H. Bagci, and E. Michielssen, "An adaptive multi-element probabilistic collocation method for statistical emc/emi characterization," Electromagnetic Compatibility, IEEE Transactions on, vol. 55, no. 6, pp. 1154-1168, Dec 2013.

14. S. Skelboe, "True worst-case analysis of linear electrical circuits by interval arithmetic," Circuits and Systems, IEEE Transactions on, vol. 26, no. 10, pp. 874-879, Oct 1979.

15. W. Tian, X.-T. Ling, and R. Liu, "Novel methods for circuit worst-case tolerance analysis," Circuits and Systems I: Fundamental Theory and Applications, IEEE Transactions on, vol. 43, no. 4, pp. 272-278, Apr 1996.

16. A. Abderrahman, E. Cerny, and B. Kaminska, "Worst case tolerance analysis and clp-based multifrequency test generation for analog circuits," Computer-Aided Design of Integrated Circuits and Systems, IEEE Transactions on, vol. 18, no. 3, pp. 332-345, Mar 1999.

17. Ferber, M.; Vollaire, C.; Krahenbuhl, L.; Vasconcelos, J. A., "Adaptive Unscented Transform for Uncertainty Quantification in EMC Large-Scale Systems," Compel (Bradford), vol.33, iss.3, pp.914-926, 2014.

18. N. Femia and G. Spagnuolo, "True worst-case circuit tolerance analysis using genetic algorithms and affine arithmetic," Circuits and Systems I: Fundamental Theory and Applications, IEEE Transactions on, vol. 47, no. 9, pp. 1285-1296, Sep 2000

19. Sami Barmada, Antonino Musolino, Marco Raugi and Mauro Tucci, "A delta-method technique in the wavelet domain to determine statistical quantities of the response of electromagnetic devices with uncertain parameters" in Int. J. Numer. Model. 2011; 24:357-374.

20. Ferber, M.; Korniienko, A.; Scorletti, G.; Vollaire, C.; Morel, F.; Krahenbuhl, L., "Systematic LFT Derivation of Uncertain Electrical Circuits for the Worst-Case Tolerance Analysis," in IEEE Transactions on Electromagnetic Compatibility, vol.57, iss.5, pp.937-946, 2015.

21. S. Boersma, A. Korniienko, K. Laib, J.W. van Wingerden, Robust Performance Analysis for a Range of Frequencies, IEEE American Control Conference, Boston, USA, July 2016, pp. 5664 - 5670.

22. J. Doyle, A. Packard, and K. Zhou, "Review of LFTs, LMIs, and $\mu$," in Decision and Control, 1991., Proceedings of the 30th IEEE Conference on, Dec 1991, pp. 1227-1232 vol.2.

23. Johan Lofberg, "YALMIP: A toolbox for modeling and optimization in MATLAB" in Computer Aided Control Systems Design, 2004 IEEE International Symposium on, 2004, pp.284-289.

24. MOSEK ApS, 'The MOSEK optimization toolbox for MATLAB manual. Version 7.1 (Revision 28)," in 2015.

25. MATLAB linmod, Online: http://www.mathworks.com/help/simulink/slref/linmod.html.

26. J. Doyle, ”Analysis of Feedback Systems with Structured Uncertainties", IEEE Proceedings, Nov 1982, pp. 242-250, vol 129-D, 6.

27. M. K. H. Fan and A. L. Tits and J. C. Doyle, "Robustness in the presence of mixed parametric uncertainty and unmodeled dynamics", IEEE Transaction on Automatic Control, Jan 1991, pp. 25-38, vol 36, 1. 